\title{
FLASHLIGHT: \\ Fluorescent Lyman-Alpha Survey of cosmic Hydrogen iLlumInated by hIGH-redshifT quasars.
}

\author{
Fabrizio Arrigoni Battaia ${ }^{1}$, J. F. Hennawi ${ }^{1}$, \\ S. Cantalupo ${ }^{2,3}$ and J. X. Prochaska ${ }^{2,3}$ \\ ${ }^{1}$ Max-Planck-Institut für Astronomie, \\ Königstuhl 17, D-69117 Heidelberg, Germany \\ email: arrigoni@mpia.de \\ ${ }^{2}$ Department of Astronomy and Astrophysics, University of California, \\ 1156 High Street, Santa Cruz, CA, 95064, USA \\ ${ }^{3}$ University of California Observatories, Lick Observatory, \\ 1156 High Street, Santa Cruz, CA, 95064, USA
}

\begin{abstract}
The current galaxy formation paradigm postulates that a significant amount of gas accretes onto galaxies from the IGM in a cold $T \sim 10^{4} \mathrm{~K}$ phase, and many have argued that this gas should be detectable in diffuse Ly $\alpha$ emission. However, so far, there are no convincing direct detections.

We are conducting a deep (down to $1 \sigma \sim 5-10 \times 10^{-19} \mathrm{erg} \mathrm{s}^{-1} \mathrm{~cm}^{-2} \operatorname{arcsec}^{-2}$ ) and the first statistical survey matching the $\operatorname{Ly} \alpha$ line around radio-quiet quasars at $z \sim 2$. By taking advantage of the boost in fluorescent Ly $\alpha$ emission due to the ionizing radiation emitted by a luminous QSO that, like a flashlight, can illuminate the hydrogen in its vicinity, we will be able to constrain some of the properties of the gas sorrounding $z \sim 2$ QSOs. In particular, with this unique dataset, we can conduct the first deep statistical census of the size, morphology, luminosity, frequency, and covering factor of Ly $\alpha$ emission around QSOs.
\end{abstract}

Keywords. quasars, intergalactic medium, halos, fluorescence

\section{Introduction}

Until recently, the accepted model for galaxy formation was that baryons collapsed onto dark matter halos, were shock-heated to the virial temperature, and subsequently cooled to low temperatures to form stars (White \& Rees 1978). However, it is now clear that cooling from a hot virialized halo accounts for only a small fraction of the fuel for star formation (Kereš et al. 2005, Ocvirk et al. 2008). Instead, cosmological hydrodynamical simulations indicate that a 'cold mode' of accretion onto galaxies occurs along filaments. The most attractive feature of this now-ubiquitous picture (e.g., Cattaneo et al. 2007) is the efficient transport of cold gas $\left(T \sim 10^{4} \mathrm{~K}\right)$ to the centers of $z \sim 2-3$ starforming galaxies, providing a natural mechanism to sustain large star formation rates at high-redshift (Solomon \& Vanden Bout 2005, Erb 2008). However, direct observational evidence for the presence of these cold flows is thus far non-existent.

Because the Ly $\alpha$ line is the primary coolant of cold $T \sim 10^{4} \mathrm{~K}$ low metallicity gas, a plethora of simulation studies have argued that $\mathrm{Ly} \alpha$ 'cooling radiation' could be detectable from this cold flow gas as it accretes onto halos (e.g. Haiman et al. 2000, Fardal et al. 2001, Frank et al. 2012). 
Here we focused on QSO environments, where the luminous QSO, acting like a flashlight, illuminate with its ionizing radiation the sorrounding hydrogen gas. The photoionized gas will than produces Ly $\alpha$ recombination photons, which will enhance the ubiquitous fluorescence signal due to the UV background, and in general will boost the signal from the cold gas. Thus, this process could allow us to detect this cold flow gas even if the cooling radiation itself is too faint. Therefore, the Ly $\alpha$ photons that escape the system, will inform us about the physical properties of the emitting gas.

This idea is now becoming well established in the literature. Starting from the theoretical expectations by Hogan \& Weymann (1987), and later by e.g. Binette et al. (1993), Gould \& Weinberg (1996), Cantalupo et al. (2005), to the numerous observational efforts for detecting the fluorescent signal from the UV background (e.g. Lowenthal et al. 1990, Rauch et al. 2008), and around quasars (e.g. Fynbo et al. 1999, Adelberger et al. 2006). However, all of these attempts yelded either null or questionable detections.

The situation changed with a recent pilot survey by Cantalupo et al. (2012), who proved the feasibility of detecting the fluorescence signal with the current facilities. In particular, using a custom-built filter on FORS/VLT, they carried out a deep narrowband search for Ly $\alpha$ emission around the hyperluminous quasar HE0109-3518 at $z=2.4$, probing a volume of 5500 comoving $\mathrm{Mpc}^{3}$ down to their flux limit $\left(\mathrm{F}_{L y \alpha} \sim 4 \times 10^{-} 18 \mathrm{erg} \mathrm{s}^{-1} \mathrm{~cm}^{-2}\right)$. They detected a hundred of Ly $\alpha$ candidates, 12 of which are the most convincing candidates to date for fluorescent emitters, showing a rest-frame equivalent width larger than $240 \AA$, the maximum predicted value for internal star formation (Charlot \& Fall 1993).

Our survey is the natural continuation of the same experiment, focusing more on the close vicinity of the QSO, on the circum galactic medium (CGM), where recent absorption studies have shown the presence of massive $\left(\mathrm{M}_{C G M}>10^{10} \mathrm{M}_{\odot}\right)$ cold gas $\left(T \sim 10^{4} \mathrm{~K}\right)$ with high covering factor (Prochaska et al. 2013a, Prochaska et al. 2013b). Thus, as our technique will allow us to put constraints on the emission from the CGM gas, our work will be complementary to the absorption-line studies recently carried out (Hennawi \& Prochaska 2013) and will provide a fundamental benchmark for future observations with new instruments.

\section{Overview}

The survey is actually conducted using the LRIS instrument on the 10m Keck-I telescope and GMOS-S on the $8.1 \mathrm{~m}$ Gemini South telescope. P. I. of these observations are respectively J. X. Prochaska and S. Cantalupo.

As the signal we are looking for is at the limit of the current facilities, we are using the following highly optimized approach. We have purchased a set of custom narrowband filters, tuned at the Ly $\alpha$ line of $z \sim 2$ radio-quiet QSOs, with the narrowest bandpass possible $(\sim 30-40 \AA)$ that allows a good throughput $(\geqslant 50 \%)$. In addition, the central wavelength of these filters corresponds to minima in the sky background, and all the observations are carried out in dark periods to avoid the increased blue background from the moon.

All the above requirements makes our approach more efficient than previous searches, which used wider filters $(70-100 \AA$ A) (e.g. Bergeron et al. 1999) and imaged object at higher redshift (e.g. Christensen et al. 2006), where the $(1+z)^{4} \mathrm{SB}$ dimming makes harder to achieve the depth required to detect the fluorescence signal, i.e. to achieve the same depth for a $z \sim 3$ QSO one must integrate $\sim 10$ times longer than at $z \sim 2$.

Further, owning to the presence of a dichroic, the LRIS instrument allow us to simultaneously take the narrowband image and a redder broad-band image (e.g. $V$-band) used 


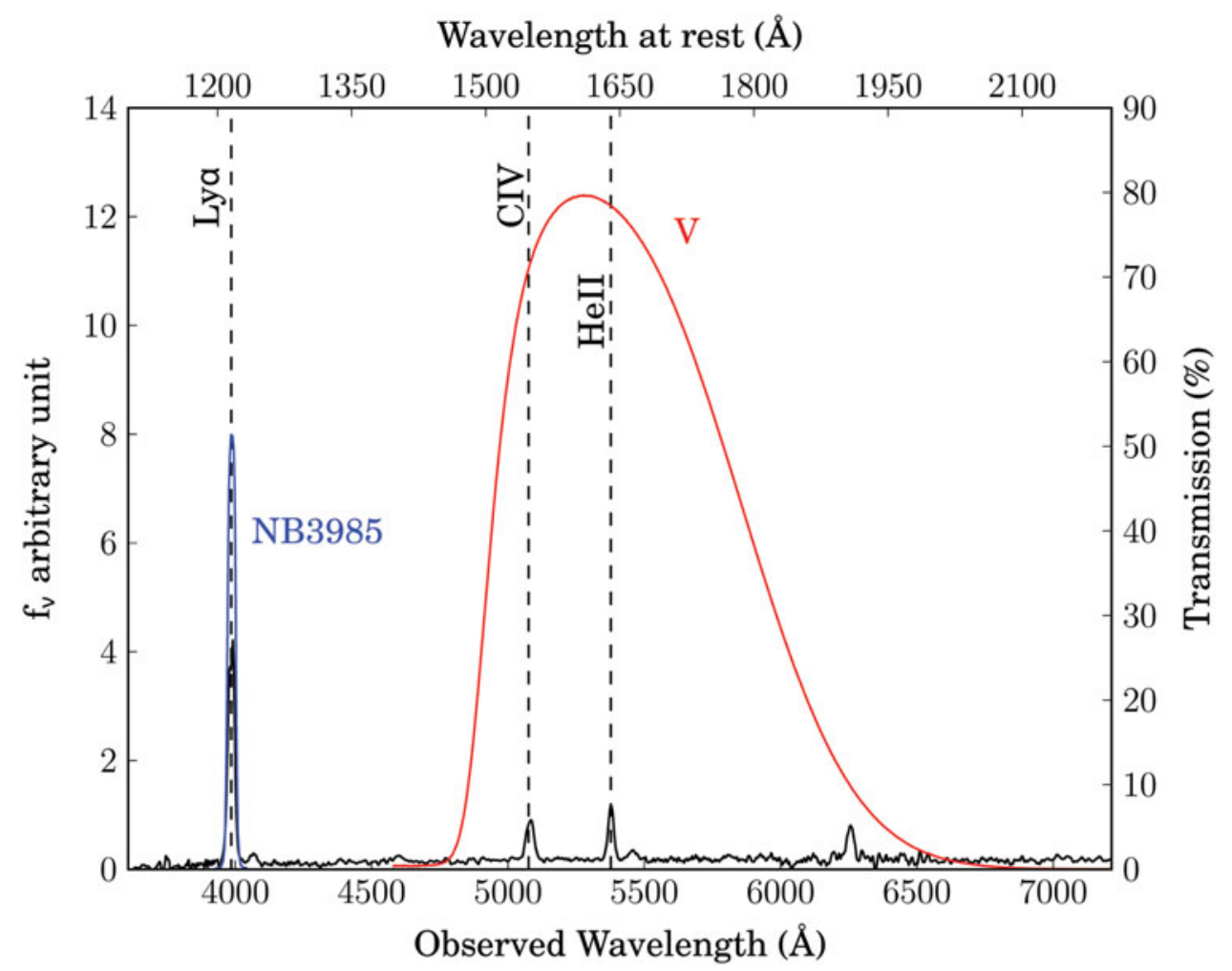

Figure 1. Example of a filter configuration in our survey, while using the LRIS instrument on the Keck-I telescope. The plot shows the bandpass for our custom narrowband filters NB3985 (blue), and the broad-band filters $V$ (red). In this case, the narroband filter is matching the Ly $\alpha$ at $z \sim 2.278$.

to determine the continuum. In this way our program is faster in terms of telescope time. See Figure 1 to see a typical filter configuration used in our survey.

\section{Preliminary Results}

Due to publications in high-profiles journals, the results shown at the Symposium cannot be included here. The anticipated date of publication of those results is end of this year or early 2014.

Papers describing the complete survey and its results will appear during next years.

The same dataset will be used also for constraining the population of fluorescent objects at Mpc distances from the QSO by S.Cantalupo and collaborators.

\section{References}

Adelberger, K. L., Steidel, C. C., Kollmeier, J. A., \& Reddy, N. A. 2006, ApJ, 637, 74

Bergeron, J., Petitjean, P., Cristiani, S., et al. 1999, A\& A, 343, L40

Binette, L., Wang, J. C. L., Zuo, L., \& Magris, C. G. 1993, AJ, 105, 797

Cantalupo, S., Porciani, C., Lilly, S. J., \& Miniati, F. 2005, ApJ, 628, 61

Cantalupo, S., Lilly, S. J., \& Haehnelt, M. G. 2012, MNRAS, 425, 1992

Cattaneo, A., Blaizot, J., Weinberg, D. H., et al. 2007, MNRAS, 377, 63

Charlot, S. \& Fall, S. M. 1993, ApJ, 415, 580

Christensen, L., Jahnke, K., Wisotzki, L., \& Sanchez, S. F. 2006, A\& A, 459, 717 
Erb, D. K. 2008, ApJ, 674, 151

Fardal, M. A., Katz, N., Gardner, J. P., et al. 2001, ApJ, 562, 605

Frank, S., Rasera, Y., Vibert, D., et al. 2012, MNRAS, 420, 1731

Fynbo, J. U., Møller, P., \& Warren, S. J. 1999, MNRAS, 305, 849

Gould, A. \& Weinberg, D. H. 1996, ApJ, 468, 462

Haiman, Z., Spaans, M., \& Quataert, E. 2000, ApJ (Letters), 537, L5

Hennawi, J. F. \& Prochaska, J. X. 2013, ApJ, 766, 58

Hogan, C. J. \& Weymann, R. J. 1987, MNRAS, 225, 1P

Kereš, D., Katz, N., Weinberg, D. H., \& Davé, R. 2005, MNRAS, 363, 2

Lowenthal, J. D., Hogan, C. J., Leach, R. W., Schmidt, G. D., \& Foltz, C. B. 1990, ApJ, 357, 3

Ocvirk, P., Pichon, C., \& Teyssier, R. 2008, MNRAS, 390, 1326

Prochaska, J. X., Hennawi, J. F., \& Simcoe, R. A. 2013a, ApJ (Letters), 762, L19

Prochaska, J. X., Hennawi, J. F., Lee, K.-G., et al. 2013b, ApJ, 776, 136

Rauch, M., Haehnelt, M., Bunker, A., et al. 2008, ApJ, 681, 856

Solomon, P. M. \& Vanden Bout, P. A. 2005, ARAA, 43, 677

White, S. D. M. \& Rees, M. J. 1978, MNRAS, 183, 341 